Relations industrielles

Industrial Relations

\title{
Longer Life. By George H. Soule. New-York: The Viking Press,
} 1958. 151 pp. \$3.00.

\section{Roger Chartier}

Volume 15, numéro 4, octobre 1960

URI : https://id.erudit.org/iderudit/1021956ar

DOI : https://doi.org/10.7202/1021956ar

Aller au sommaire du numéro

Éditeur(s)

Département des relations industrielles de l’Université Laval

ISSN

0034-379X (imprimé)

1703-8138 (numérique)

Découvrir la revue

Citer ce compte rendu

Chartier, R. (1960). Compte rendu de [Longer Life. By George H. Soule. New-York: The Viking Press, 1958. 151 pp. \$3.00.] Relations industrielles / Industrial Relations, 15(4), 518-519. https://doi.org/10.7202/1021956ar

Tous droits réservés @ Département des relations industrielles de l’Université Laval, 1960
Ce document est protégé par la loi sur le droit d'auteur. L'utilisation des services d'Érudit (y compris la reproduction) est assujettie à sa politique d'utilisation que vous pouvez consulter en ligne.

https://apropos.erudit.org/fr/usagers/politique-dutilisation/ 
Voici un petit ouvrage sur certains aspects de la réalité industrielle japonaise qui invite les Occidentaux que nous sommes à une réexamen du concept d'efficacité dans l'industrie.

Le développement industriel phénoménal du Japon, qui dépasse de beaucoup en rapidité et en ampleur celui des autres pays asiatiques, pose un gros point d'interrogation aux industriels et aux théoriciens (sociologues et économistes en particulier). Pourquoi, en somme, les Japonais ont-ils si bien assimilé et adapté à leurs fins la technologie industrielle de l'Occident, alors que tant d'autres nations africaines ou asiatiques éprouvent des difficultés culturelles si grandes à absorber le processus de l'industrialisation?

D’après les économistes classiques et les théoriciens-sociologiques comme Marshall, Pareto, Sombart, Weber, Toennies et combien d'autres, la société industrielle moderne se caractérise avant tout par la rationnalité des relations économiques en général, de l'organisation des entreprises et des techniques de production. Puisque donc tout et tous dans l'industrie sont si raisonnables, il devrait être facile de «motiver» les travailleurs économiquement; car, en bonne logique, ces derniers sont libres de poursuivre leurs intérêts économiques « rationnellement », d'acheter biens et services à meilleur marché, de se déplacer professionnellement et géographiquement, de signer les contrats les plus avantageux; de même, la direction sera libre d'embaucher ou de congédier son personnel au gré de ses besoins «raisonnables». On connaît d'ailleurs la thèse de Weber sur la bureaucratie, assise sur la compétence technique, illustrée par la hiérarchisation des positions, impersonnelle et efficace.

Or, la réalité japonaise que nous découvre Abegglen s'accommode fort mal de ces schèmes théoriques. L'auteur observe de près plus de cinquante entreprises industrielles nippones. Il y fait, en gros, les «découvertes» suivantes: 1) l'entreprise japonaise se sent responsable de son personnel bien au delà des strictes relations de production; 2) la rémunération du personnel n'est que partiellement monétaire; elle se fonde sur des critères sociaux généraux plutôt que sur des critères de production: l'âge, l'instruction, l'ancienneté et le nombre d'enfants prennent souvent le pas sur des considérations économiques de rendement; 3) la tâche d'un individu a un véritable caractère de permanence; au Japon, on trouve peu de congédiements, de mises à pied, de mobilité géographique; 4) la structure formelle de l'entreprise est très élaborée; les décisions s'y prennent collectivement, et les responsabilités sont collectives plutôt qu'individuelles; 5) la position (《status 》) à l'usine est le prolongement de la position sociale globale; et 6 ) le recrutement s'appuie moins sur les exigences objectives de la tâche que sur certaines qualités personnelles d'instruction, de caractère et d'arrière-plan social ou économique.

C'est dire que le Japon s'est donné une structure industrielle adaptée à ses valeurs sociales, et notamment familiales. De sorte que ce que le système perd en rationnalité, en flexibilité et en « efficacité », il le gagne en stabilité, en solidarité, en loyauté et en adaptation plus facile au mode de vie industriel.

Le livre est fort bien écrit et très instructif par les comparaisons qu'il suggere et par l'obligation où il place les Occidentaux de reconnaître la relativité de leurs éléments culturels et de leurs thèses sur les impératifs de l'organisation et du développement industriels.

\section{Roger Chartier}

Longer Life. By George H. Soule. New York: The Viking Press, 1958. $151 \mathrm{pp}$. $\$ 3.00$.

Voici un tout petit livre qui résume admirablement bien, dans une langue claire et simple, les connaissances acquises jusqu'ici en matière de gérontologie sociale et économique. Rien, en fait, qui fasse avancer la science du vieillissement des humains, mais beaucoup pour la vulgariser.

L'auteur aligne les causes les plus courantes de la mort, définit expérimentalement le vieillissement, souligne les aptitudes relatives ( au travail et ailleurs) des personnes qui gagnent en maturité, leurs difficultés économiques, leurs tribulations comme personnes à charge, la ségrégation dont ils sont l'objet et la nécessité sociale de leur réintégration.

En bon économiste, l'auteur aborde assez longuement les aspects économi- 
ques des pensions et des formules de retraite, au plan de l'individu comme à celui de la nation. Soule se refuse à trouver avantageuse, à quelque point de vue que ce soit, la retraite obligatoire à un âge fixé arbitrairement; celle-ci diminue le nombre de travailleurs productifs ainsi que le niveau de leur revenu et du revenu national. Selon l'auteur, un pays ne peut se permettre une classe taite de millions d'inactifs qui sont hostiles à la retraite pour toutes sortes de raisons, psychologiques, sociologiques et financières.

D'autre part, il importe de ménager aux travailleurs âgés qui ne peuvent plus trouver à s'employer ou qui ne souhaitent plus continuer leur vie économiquement active des revenus plus considérables pour qu'ils puissent vraiment jouir de leur retraite.

Toutes les données de la science médicale tendent dans le même sens: la durée moyenne de vie s'allonge, la santé des personnes mûres s'accroît sans cesse. Il faut donc cesser d'attacher comme une tare, une évaluation pathologique à quiconque dépasse tel niveau d'âge. Il faut rétablir l'équilibre entre ces travailleurs de l'industrie et du commerce qui n'ont présentement pas suffisamment de loisirs, et les vieux ex-travailleurs à qui on en impose trop.

\section{Roger Chartier}

Relations humaines et relations industrielles. Par Marcel Bolle de Bal. Bruxelles: Institut de Sociologie Solvay, 1958. $135 \mathrm{pp}$.

Plusieurs auteurs américains, avec Harbison \& Coleman ( $L a$ négociation collective), ont décrit en termes quelque peu interrogatifs la \& zone neutre et imprécise des relations humaines ». Les « human relations », comme champ d'étude et comme optique dans l'industrie, sont une réalité décidément nord-américaine, aux contours plutôt flous, qui enjambe prestement et confond souvent les schèmes des diverses sciences de l'homme, et notamment la sociologie et la psychologie.

Le concept de «relations humaines», en bref, a nettement besoin de clarification. Car il recouvre tour à tour un fait social et une politique industrielle, un objet d'études et un ensemble de tech- niques psycho-sociologiques, un facteur de productivité et un état d'esprit. $\mathrm{Pa}$ reille imprécision dans les perspectives ne manque pas d'éveiller des inquiétudes chez les chercheurs du social comme chez les praticiens de l'industrie, patrons et chefs syndicaux. De sorte que la chasse à l'équivoque est doublement importante dans ce domaine où valeurs et intérêts s'entrechoquent.

Il est heureux, en conséquence, que des éclaircissements soient fournis sur les « relations humaines», et que de plus ils nous viennent d'un Européen qui a étudié sur place la réalité industrielle américaine.

Dans ce petit livre sans prétention, l'auteur analyse les divers aspects des relations humaines. Distinguant le plan de la recherche théorique et celui de l'action pratique, il dresse un bilan rétrospectif des études et des politiques de «human relations ». Il montre les promesses et les dangers des unes et des autres, soulignant les écueils d'une «fausse science» et d'une «fausse démocratie » industrielles. Il fait le joint entre «relations humaines », productivité et harmonie dans l'industrie.

S'appuyant sur certaines expériences réalisées dans l'industrie belge, l'auteur dégage les causes d'échec et les facteurs de réussite en matière de relations du travail.

Voici donc un petit ouvrage-synthèse qui rendra de grands services.

\section{Roger Chartien}

\section{«Technological Changes and Skilled Manpower: The Automobile and Parts Manufacturing Industries », Depart- ment of Labour, Canada, in co-opera- tion with federal and provincial government agencies and other groups, September 60, 54 pp.}

Le ministère fédéral du travail vient de publier la $8 \mathrm{e}$ d'une série d'études concernant l'entraînement de la maind'oeuvre spécialisée au Canada. On sait que le ministère, conjointement avec d'autres organismes gouvernementaux aux niveaux fédéral, provincial et municipal, entreprenait dès 1956 une vaste série d'enquêtes à ce sujet. La présente étude traite des effets des changements 